\title{
THE INFORMATION REQUIREMENTS OF COMPLEX BIOLOGICAL AND ECONOMIC SYSTEMS WITH ALGORITHMIC INFORMATION THEORY
}

\author{
S. DEVINE \\ Victoria Management School, Victoria University of Wellington, Wellington, New Zealand.
}

\begin{abstract}
This article interprets the natural laws creating and maintaining a complex system, such as an ecology or an economy, distant from equilibrium, as computations on a real world Universal Turing Machine (UTM). As a laboratory, UTM can simulate the real world UTM, from the perspective of algorithmic information theory, the number of bits in the shortest, appropriately coded binary algorithm that specifies a real world system on a laboratory UTM defines its algorithmic entropy and its information content. As only algorithmic entropy differences matter, and differences are UTMindependent, differences measured on the laboratory UTM align with entropy changes in the real world. The system's distance from equilibrium in bits defines its order. Computations require energy. Landauer's principle identifies the minimum energy per bit (or the real world equivalent) to drive the computation that creates and sustains a real world system in a homeostatic state distant from equilibrium. This high-grade energy carries the computational instructions that do work on the system, ejecting disorder as heat and waste. While replication algorithms drive the emergence of complex ecological systems (doi:10.1016/j.biosystems.2015.11.008), in economic systems, individual agent behaviour can be captured by computer algorithms akin to the perspective of an adaptive system paradigm. Rather than specifying detailed behavioural routines for an economy, a narrative is used to identify the information drivers that create an ordered far-from-equilibrium economic system. The narrative shows that, somewhat like the interdependence of species in a vibrant ecology, agents trade, utilise technology, and amalgamate to form more complex structures creating order and driving the economy further from equilibrium. An ordered economy is a better economy. Order-creating investments (infrastructure, machines etc.) enhance economic performance, in contrast to non-ordering investments that extract wealth from others, adding nothing.

Keywords: Algorithmic Information Theory, economic complexity and economic order, emergence, energy and economic sustainability, non-equilibrium economics.
\end{abstract}

\section{INTRODUCTION}

Here it is argued from the Algorithmic Information Theory (AIT) that the order observed in a complex natural system emerges through processes that create and maintain the system distant from thermodynamic equilibrium. AIT defines the Kolmogorov or algorithmic complexity of a structure by the number of bits in the shortest binary string that specifies the structure and then halts (e.g. see [1]). Importantly, when appropriately coded, the number of bits defines the algorithmic entropy that is related to the traditional entropies. AIT has been used to track the emergence of order in biological systems [2], but conceptual issues need to be explored to effectively apply the approach more widely.

\subsection{Characteristics of an ordered complex system from an AIT perspective}

This article shows that a complex living system, such as an ecology or an economy, is highly ordered because it is distant from its local thermodynamic equilibrium. Here, the local equilibrium identifies the most disordered state of the natural system that emerges over time, once sustaining energy sources are removed. From an AIT perspective, the detailed state of such 
an ordered system can be described by a shorter algorithm than one that describes the local equilibrium state. The complexity that manifests itself in an ordered natural system is seen to be of consequence to the processes that create and maintain the system distant from its local equilibrium. The order emerges when natural processes harness stored energy to re-configure the system and to eject waste. Importantly, the natural laws creating and maintaining order can be seen as equivalent to computational processes implemented on a real world Universal Turing Machine (UTM), i.e. a real world general computer. But the computations on a real world UTM can be simulated to within a machine-dependent constant by a laboratory UTM $[1,3]$. This understanding allows the order to be recognised because an ordered system can be specified using a laboratory UTM with fewer programme bits than a disordered one. But, as is discussed further in Section 2, information differences are independent of the UTM used. The flow of information (converted to bits) passing through a real world computation is the same as the laboratory simulation [2]. As a consequence, if $H(s)$ is the number of programme bits to describe the system, and $H($ equil) is the number of bits to describe its disordered local equilibrium, the degree of order is $H($ equil $)-H(s)$. This is the information defining the ordered system and is UTM-independent.

\subsubsection{Natural ordering processes}

One bacterium immersed in a flow of nutrients, will reproduce by harnessing the energy in the nutrients, until the number of bacteria reach the system's carrying capacity, as determined by the efficiency of the process and the rate of flow through of nutrients. At the carrying capacity, the system can be specified by a short algorithm consisting of a routine that specifies one bacterium and which is called many times. On the other hand, if the nutrient flow is stopped, the system will revert to a disordered local equilibrium of randomly distributed chemicals. The disordered equilibrium can only be specified by an horrifically long algorithm. Order has been created by natural replication that, as computational processes, access programme information in the nutrients to reproduce and eject waste and heat. Similarly, an economy of hunter gatherers with restricted food supplies will spin out new family units until the system reaches its carrying capacity. Again, the system that emerges is more ordered than the local equilibrium that appears when the programme information carried in food, is no longer available.

A complex system in the natural world can be envisaged as a computational system, where the physical laws act as programmes stored in the energy states defining the system. Order is created and, once created, can be maintained as the system trends to a homeostatic or stable state distant from equilibrium. Computational processes are implemented using the information stored as high grade, energy, (i.e. in the nutrients) to create or re-create order, ejecting disorder as waste and heat. As the ordered complex systems that emerge through replication processes show pattern, they can be described by an algorithm much shorter than a disorganised system. Their degree of order is higher.

\section{THE AIT APPROACH}

AIT was originally conceived by Solomonoff [4], and developed by Kolmogorov [5] and Chaitin [6]. Its application to the natural world is based on the recognition that the instantaneous configuration, or microstate, of a natural system can be systematically defined in an appropriate state space by a binary string of digits, denoted by $s_{i}$, AIT defines the information content of the system as the length of the shortest algorithm run on a UTM that generates $s_{i}$ and then halts. This length is also known as the Kolmogorov complexity, [5], or the 
algorithmic complexity [1, 3]. Critically, as instructions implemented on the real world computer are processed instantaneously, they are self-delimiting. Consequently, if the laboratory UTM uses self-delimiting instructions $[1,3]$, the information measure of string $s_{i}$ representing the living system is called the algorithmic entropy and is denoted by $H\left(s_{i}\right)$.

The algorithmic entropy aligns with the Shannon entropy and the entropy of thermodynamics $[2,7,8]$. Whenever pattern or structure is observed in a string defining a natural system, an algorithm can be constructed to provide a compressed description of the string, although unrecognised structure may allow further compression. For example, a simple algorithm can generate the first million digits of $\pi$, but a random string of a million digits requires each digit to be specified.

\subsection{Algorithmic specification of a system}

The AIT approach can be demonstrated by considering the shortest algorithm that can specify an image of 1 million pixels where ' 0 ' represents a white pixel and ' 1 ' represents a black pixel. The algorithm that specifies a random array of pixels is of the following form, as each pixel must be separately specified.

$$
\text { OUTPUT'1001101. . .1100'. }
$$

Here vertical lines, $\mid$. . .| denote the number of bits bracketed between the lines. The length of the algorithm, given by $H$, is

$$
H=\mid \text { OUTPUT }|+| 1001101 \ldots 1100 \mid+C .
$$

$H$ is a little more than 1 million bits, as it requires both the bits in the output instruction and the image to be specified. $C$ is the constant defining the UTM used. However, if the image shows pattern, a shorter algorithm, such as a high-resolution JPEG compression may be appropriate. A completely ordered image, where every pixel is black (or white) can be specified by an algorithm of the form:

\section{OUTPUT ' 1 ' a million times.}

In which case $H$ is close to

$$
H=\mid \text { OUTPUT }|+| 1|+| 1,000,000 \mid+C .
$$

The number of bits defining $H$ includes the length of the output instruction, the specification of ' 1 ', the specification of the number 1,000,000 and the computer constant $C$. As the second to last term is close to $\log _{2}(1,000,000)=20$ bits, an algorithm of a little more than 20 bits can generate the image. However, more than $1,000,000$ bits are needed to generate the random image. The difference between the number of bits to define the disordered image and the ordered image is the degree of order, i.e. 999,980 bits. Order is rare, as the overwhelming majority of the arrangements or configurations of the possible image will have descriptions close to $1,000,000$ bits. As only information or entropy differences are relevant, the machine dependence and common instructions cancel.

\subsubsection{The emergence of order through relatively simple computations}

While a simple algorithm can specify the instantaneous state of a real world complex system, such a system also emerges through computational processes as illustrated by Conway's Game of Life (see Wikipedia [9]). The complex structures that emerge in an interconnected 
and nested living ecology are analogous to the way small clusters of independent black pixels in the Game of Life roam through image space, and re-organise using simple rules to produce emergent structures. The rules of interaction create more ordered arrangements that are able to be defined by short algorithms, i.e. a simple algorithmic set of rules generate the system, allowing a short algorithm to specify the ordered outcome. The analogy is more relevant to the natural world if the pixels in the image example degrade by processes analogous to the second law of thermodynamics, i.e. let black pixels randomly decay to white pixels as time flows. In order to reverse such a degradation, a programme carrying energy must regenerate a degraded pixel, and eject the heat released when a black pixel becomes a white pixel.

\subsection{Landauer's principle}

The bits in the programme that define a real world system carry energy. Real world processes are computations driven by programmes embodied in energy levels of natural species that act as the equivalent of gates. Landauer's principle [10] identifies the minimum energy associated with each programme bit (or the real world equivalent) [8, 11-13]. The energy, but not the information, depends on the computational temperature. The principle states that, where one bit of information in a computational machine operating at a temperature $T$ is erased, at least $k_{B} T \ln 2$ Joules must be dissipated. When the length of the algorithmic description of the state of a system changes as $\Delta H$ bits are added or removed, the corresponding thermodynamic entropy change in the real world system is $k_{B} \ln 2 \Delta H$. The validity of Landauer's principle has been discussed by Leff and Rex [14] and has been experimentally verified [15-17].

\subsection{The provisional entropy of a set of states}

A real world ecology usually settles in a stable set of configurations or microstates defined by variables such as temperature, pressure or energy, and the number of species that define the homeostatic macrostate distant from the local equilibrium. The neo-classical economic concept of equilibrium, where all economic forces balance, is seen to correspond to a homeostatic macrostate once energy is taken into account. The corresponding microstates are the myriad of allowable internal arrangement. All the instantaneous microstates, belonging to the same macrostate, exhibit a common structure, just as the particular microstate of a simple gas is specified by the instantaneous position and the momentum of each particle. If there are $\Omega$ microstates in the macrostate, a particular microstate, can be specified by a two-stage algorithm. The first identifies the particular microstate in the macrostate and has length $\log _{2} \Omega$ bits, while the second defines the characteristics of the set of allowable microstates $[2,18]$. The information content that is common to each microstate in the macrostate is called the provisional entropy $H_{\text {prov }}$ and is given by [18]:

$$
H_{\text {prov }}\left(s_{i}\right)=\log _{2} \Omega+H(\text { specification of macrostate set })
$$

This measure is similar to the Gács entropy, Kolmogorov's Algorithmic Minimum Sufficient Statistic and Zureks intuitive physical entropy concept [2]. The entropy of Shannon's information theory, by contrast, is a characteristic of the macrostate itself and corresponds to $H($ Shannon $)=\log _{2} \Omega$. The two entropies are related by

$$
H_{\text {prov }}\left(s_{i}\right)=H(\text { Shannon })+H(\text { specification of macrostate set }) \text {. }
$$


Because the provisional entropy also requires the characteristics of the macrostate, or set of states to be specified, it requires more bits than does the Shannon entropy. For an equilibrium set of states, they are virtually the same, i.e. $H_{\text {prov }}($ equil $)=H($ Shannon $)$.

\subsection{Residual information replaces snapshot entropy}

As the algorithm that provides a snapshot of the information defining the system must halt, the algorithmic entropy aligns with the traditional entropies. But real world computations (as illustrated by the computations generating the ordered bacterial system) are on-going. The system settles in the homeostatic state determined by the carrying capacity. While information flows in and out, moving the system from one microstate to another, the number of bits, determined by the provisional entropy is conserved. The provisional entropy, which is the number of residual bits in the system, equals the initial number of bits, plus the bits that flow into the system, less the bits that flow out [2]. The bit flows that are a consequence of the on-going real world computations, can be tracked to determine the residual or provisional entropy. Landauer's principle relates the residual bits in the on-going computations to the energy that characterises the system at temperature $T$.

\subsection{A simple example of a real world computational system}

The computational processes that form water when $\mathrm{H}_{2}$ and $\mathrm{O}_{2}$ ignite illustrate the ideas. The programmes carried by the $\mathrm{H}_{2}$ and $\mathrm{O}_{2}$ initiate a computation that can be simulated by a laboratory UTM. Consistent with the second law of thermodynamics, the computations drive the system towards the equilibrium macrostate that consists mainly of water and heat. While the system is reversible in principle when isolated, because the overwhelming majority of states corresponds to the equilibrium set, reversibility is unlikely to occur in normal time frames. If heat is removed to return to the pre-ignition temperature, the system condenses to the more ordered state of water. The computational processes are as follows:

- Programme information in the stored energy of the hydrogen and oxygen molecules drives the system towards the equilibrium set of states, while the energy carried in the programme is diffused as heat.

- Until the heat is removed, no ordering takes place. Order requires the programme information to restructure the system and the waste from this process, in this case heat, to be removed. From an algorithmic point of view, information enters the system in the form of stored energy, and information is removed as heat to be transferred to a colder environment.

In contrast to the complexity that can be sustained by bacterial replication, the hydrogen oxygen system cannot self-sustain without an external intervention.

\section{NATURAL PROCESS TO MAINTAIN A COMPLEX SYSTEM AWAY FROM EQUILIBRIUM}

How can a natural ordered system maintain itself in a homeostatic set of microstates when the second law of thermodynamics drives it towards equilibrium? The algorithmic approach indicates that to be maintained, programmes embodied in atoms and molecules must enter the system, re-arrange its structure while ejecting information as waste [2]. Consider an 
economic or an ecological system that is in a homeostatic macrostate consisting of myriads of microstates all with the same provisional entropy. When second law processes drive the system out of the macrostate, information resources embodied in programme states must enter the system to drive it back to an equivalent microstate. Generally, for living systems, the input information is in the computational properties of food and necessary minerals etc. For the economic system fuel is also an input. From a computational perspective, the number of bits that enter the system (as programme bits, resource bits and minerals to feed the programme) must equal the number of bits that leave (mainly heat bits and partially used programme bits). The instantaneous microstate in the macrostate will move to another with the same provisional entropy as there cannot be a build-up of bits in the system. The ability of the system to counter second law degradation processes depends on the rate at which the concentrated source of energy can enter the system, and the rate at which excess bits can be ejected from the system [2] The greater the rate that the system degrades, the further from equilibrium the system is likely to be in order to eject sufficient waste such as heat.

\subsection{Order through replication, variation and selection}

Section 1.1.1 identifies the role of replication processes in creating order. In a resource-rich environment, because the probability of replication increases with the number $\mathrm{N}$ of existing structures [19], replication processes trigger a chain reaction, creating order by repeating the replicated unit. Natural computations continuously extract the information in stored energy in the resource flows while ejecting disorder, until the system of replicated structure reaches its carrying capacity. Order materialises by a resource-sensitive computation using relatively short algorithm of the form "IF SUFFICIENT RESOURCES REPEAT REPLICATED STRUCTURE" [2]. In contrast to an inert system that cannot maintain itself, replication processes naturally generate and re-generate ordered structures maintaining the system away from equilibrium.

\subsection{An order-creating narrative}

A two-stage narrative illustrates the general features whereby living systems can be maintained off equilibrium. In the first stage, given a fixed flow through of resources, natural replication processes maximise order, driving the system to its carrying capacity. A system of bacteria, or a hunter gather family, illustrates how computational processes, characterised by the bit flows through the system, create order. However, variation and selection processes give rise to replicating systems better adapted to environmental pressures. Variation in biological systems arises through mutations, but variation in human systems is primarily due to learning by the economic agents. At the smallest scale, these economic agents can be envisaged as computing units operating under 'routines', analogous to the routines of Evolutionary Economics [20], or the heterogeneous expectations of the agents in the simulation of the stock market [21]. These routines codify the deliberate, or the instinctive, behaviour of agents. As natural laws can also be specified by computational routines such as 'burn wood', 'eat and digest food', or 'reproduce', etc., the computational operation of the economy involves agents calling routines that initiate the computations embodied in natural laws. Increased order emerges as variation in economic agents through learning and discovery giving rise to different routines that can better adapt to the environment, to increase the carrying capacity. Agents, who create tools, use energy to amplify the effect of human labour 
creating a more efficient economy. The agent who creates a plough that is pulled by an animal that eats grass rather than human food, allows the carrying capacity to increase as food resources are better used.

The second-stage narrative describes how previously independent replicated units, by making better use of resources, create new interconnected and nested structure. Because there is less information waste, the overall system is more stable as it is less dependent on the external pressures. A simple example is the system where the dung beetle feeds on waste from the Wildebeest. By sharing information resources, the system is a more ordered system than when where both existed independently. Figure 1 is a schematic map of a network of interconnected computational structures that indicates how routines can be organised to provide a shorter description of what would be an extremely complicated system.

Devine [2] has shown that for biological replicating species, over significant time frames, environmental pressures and diversity due to mutations enable evolutionary processes to favour interconnected structures that use information resources more efficiently. While diversity or variety makes a system less ordered, if the diversity is embodied in software such as DNA, the effect is minimal. Schneider and Kay [22] show that the more diverse an ecology, the more effectively it uses the (solar) input resources and the more degraded is the waste ejected.

Structural ordering in economic systems is based on the same principles, but selection processes favour agents that are able to adapt through discovery and learning. Independent agents, such as the peasant farmers, organise over many generations. Those who learn to

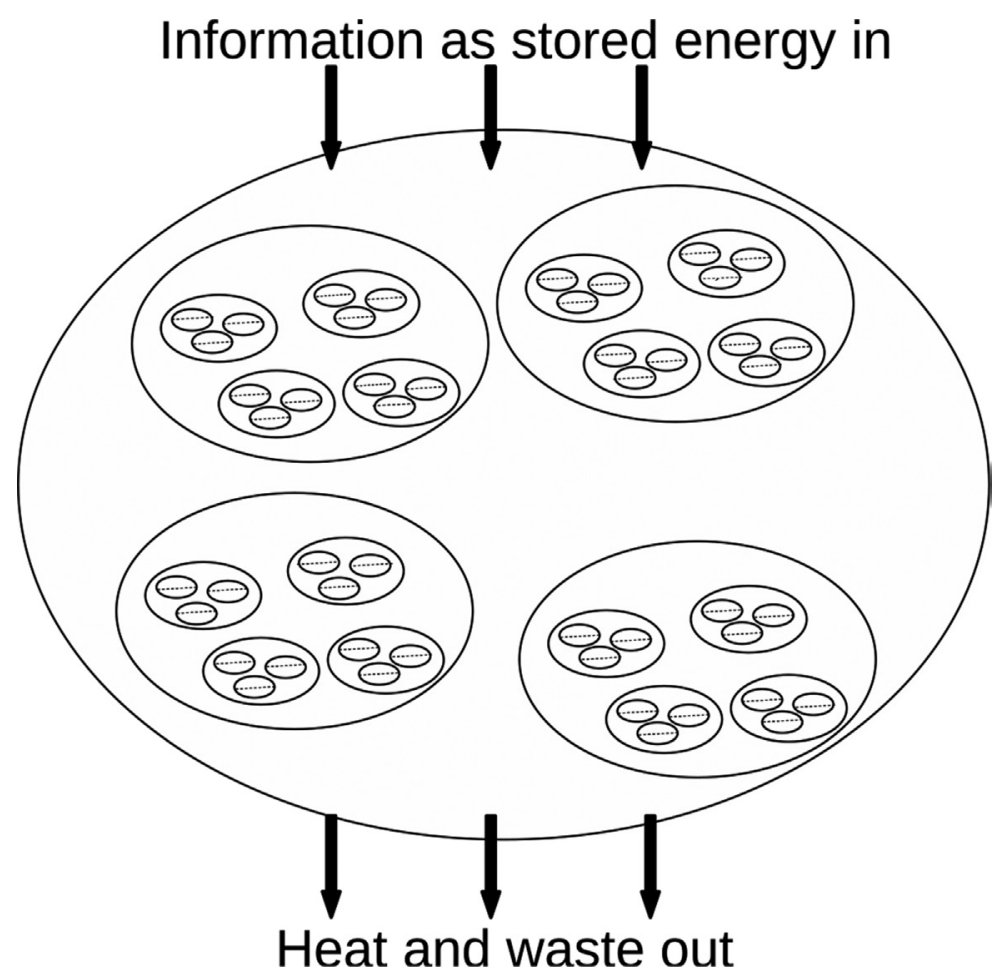

Figure 1: Nested structures. 
trade have a higher carrying capacity, as food that otherwise would be left to rot can produce more agents. The formation of tribes, firms, cities and nations are direct consequences of agents sharing resources to become interdependent and in so doing, form more ordered structures. The narrative argues that the structures and institutions, which are in effect themselves computational units, exchange information through routines directly and, in addition, call nested subroutines creating efficient super structures that are more ordered than the constituents. Cities pass information to other cities, farmers provide food containing information to cities, while within the cities, firms pass informational resources to customers and, by so doing extract the maximum benefit from these resources, while ejecting more disorder. The caveat is that the greater the order, the more energy (or information) is needed to sustain the system and the greater must be the disorder ejected in the waste.

However, somewhere in the process of economic development, behavioural routine changes. A human economy diverts its focus from maximising the carrying capacity to improving individual well-being at the cost of lower population growth. Order is still increasing, through the use of tools that use energy to enhance human labour, but the output is not ordered individuals as such, but ordered artefacts. A cell phone may be a tool that increases economic production, or an ordered artefact that increases the well-being of its owner.

\subsubsection{Reflection on economic ordering}

Because a first world economy has highly ordered collective structures and institutions, it is more ordered than one in the third world. Structural ordering has risen through the processes mentioned above where adaptive agents, by learning to use resources more efficiently, create a more ordered complex system. Observationally it appears that a more ordered economy is a better economy and there would seem to be a rough correlation between GDP and the level of order. In which case, from a strategic perspective, long-term economic investment should focus on increasing order, rather than immediate returns. It would be impossible to sustain 325 million peasant farmers or hunter gathers within a territory the size of the United States. However, in the modern United States, 325 million people can be sustained because the economy is highly ordered and structured. Nevertheless, such an ordered system is further from equilibrium than a simple peasant farmer system, and therefore is far more dependent on external energy to carry out the computational programmes that are required to create order. Critical to this has been the development of technologies that are effectively tools to amplify human labour. The human agent calls routines in the natural world to smelt iron and to produce new materials and order-creating tools. While tools themselves are ordered devices they create more order in the economy by facilitating efficient production, through accessing information embodied in stored energy in the form of coal and oil. But from an information processing perspective, the more ordered the economy the less residual information it contains, which implies that the waste from the computational processes must be thoroughly ejected. The ordering of the world's economy may well be limited by its ability to adequately reject the carbon dioxide waste from fossil fuels, which currently are accumulating in the human biosystem.

\section{CONCLUSION}

A complex living system, distant from thermodynamic equilibrium, exists in an ordered state as it can be described by a shorter algorithm than that needed to describe an equilibrium state. The degree of order, a measure of complexity, is the difference in bits between these two systems. The residual information, embodied in the ordered state is lower. The natural forces 
that drive the system away from the equilibrium create the order. A narrative to describe these processes sees a complex system of economic agents, or biological species, creating order through replication processes to expand the population to the carrying capacity. Replication is a computational process that accesses information embodied in the energy levels of resources entering a system to create order.

Other computational processes, embodied in natural processes of variation and selection, favour structures that use the computation resources entering the system more effectively, creating additional order. While variation in a biological system occurs through mutations, variation in an economic system arises through the learning processes of economic agents. Selection processes ensure that the variations using the computational resources more efficiently, will dominate the system creating structural order. Learning leads to the development of tools, and interconnections between agents such as tribes, firms, cities and nations. The order of a developed economy is much greater than a simple one, but the order needs significant energy to provide the computational resources to maintain the economy.

\section{REFERENCES}

[1] Li, M. \& Vitanyi, P.M.B., An Introduction to Kolmogorov Complexity and its Applications, 3rd edn., Springer-Verlag: New York, 2008. https://doi.org/10.1007/978-0-387-49820-1

[2] Devine, S.D., Understanding how replication processes can maintain systems away from equilibrium using algorithmic information theory. Biosystems, 140, pp. 8-22, 2016. https://doi.org/10.1016/j.biosystems.2015.11.008

[3] Chaitin, G., A theory of program size formally identical to information theory. Journal of the ACM, 22, pp. 329-340, 1975. https://doi.org/10.1145/321892.321894

[4] Solomonoff, R.J., A formal theory of inductive inference; part 1 and part 2. Information and Control, 7, pp. 1-22, 224-254, 1964.

[5] Kolmogorov, K., Three approaches to the quantitative definition of information. Problems of Information Transmission, 1, pp. 1-7, 1965.

[6] Chaitin, G., On the length of programs for computing finite binary sequences. Journal of the ACM, 13, pp. 547-569, 1966.

https://doi.org/10.1145/321356.321363

[7] Zurek, W.H., Algorithmic randomness and physical entropy. Physical Review A, 40(8), pp. 4731-4751, 1989.

https://doi.org/10.1103/PhysRevA.40.4731

[8] Bennett, C.H., Thermodynamics of computation- a review. International Journal of Theoretical Physics, 21(12), pp. 905-940, 1982.

https://doi.org/10.1007/BF02084158

[9] Conway, J., Game of life. Wikepedia, 2016.

[10] Landauer, R., Irreversibility and heat generation in the computing process. IBM Journal of Research and Development, 5, pp. 183-191, 1961.

https://doi.org/10.1147/rd.53.0183

[11] Bennett, C.H., Logical reversibility of computation. IBM Journal of Research and Development, 17, pp. 525-532, 1973. https://doi.org/10.1147/rd.176.0525

[12] Bennett, C.H., Logical depth and physical complexity. The Universal Turing Machinea Half-Century Survey, ed. R. Herken, Oxford University Press: Oxford, pp. 227-257, 1988. 
[13] Zurek, W.H., Thermodynamics of of computation, algorithmic complexity and the information metric. Nature, 341, pp. 119-124, 1989.

https://doi.org/10.1038/341119a0

[14] Leff, H.S. \& Rex, A.F., Maxwell's Demon: Entropy, Information, computing. Princeton University Press: Princeton, 1990. https://doi.org/10.1887/0750307595

[15] Berut, A., Arakelyan, A., Petrosyan, S., Ciliberto, A., Dillenschneider, R. \& Lutz, E., Experimental verification of Landauers principle linking information and thermodynamics. Nature, 483, pp. 187-190, 2012. https://doi.org/10.1038/nature10872

[16] Jun, Y., Gavrilov, M. \& Bechhoefer, J., High-precision test of Lan-dauer's principle in a feedback trap. Physical Review Letters, 113, p. 190601, 2014.

https://doi.org/10.1103/PhysRevLett.113.190601

[17] Hong, J., Lambson, B., Dhuey, S. \& Bokor, J., Experimental test of Landauer's principle in single-bit operations on nanomagnetic memory bits. Science Advances, 2(3), 2016. https://doi.org/10.1126/sciadv.1501492

[18] Devine, S.D., The insights of algorithmic entropy. Entropy, 11(1), pp. 85-110, 2009. https://doi.org/10.3390/e11010085

[19] Szathmary, E. \& Maynard Smith, J., From replicators to reproducers: the first major transitions leading to life. Journal of Theoretical Biology, 187, pp. 555-571, 1997. https://doi.org/10.1006/jtbi.1996.0389

[20] Nelson, R.R. \& Winter, S.G., An Evolutionary Theory of Economic Change. Belknap: Cambridge, MA, 1982.

[21] Arthur, W.B., Holland, J.B., Le Baron, B., Palmer, R. \& Tayler, P. (eds), Asset Pricing Under Endogenous Expectations in an Artificial Stock Market, volume XXVII of The Economy as an Evolving System II. SFI Studies in the Sciences of Complexity. AddisonWesley: Reading [MA], 1997.

[22] Schneider, E.D. \& Kay, J.J., Life as a manifestation of the second law of thermodynamcis. Mathematical and Computer Modelling, 16(6-8), pp. 25-48, 1994. https://doi.org/10.1016/0895-7177(94)90188-0 